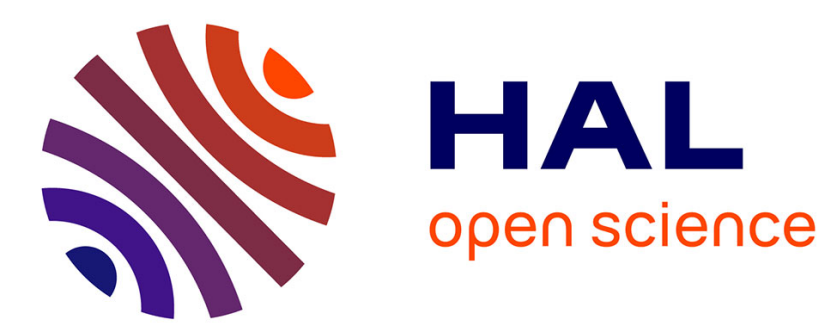

\title{
Contribution a l'étude de l'effet de magnétoconcentration. Utilité du formalisme de Boltzmann
}

\author{
S. Cristoloveanu
}

\section{> To cite this version:}

S. Cristoloveanu. Contribution a l'étude de l'effet de magnétoconcentration. Utilité du formalisme de Boltzmann. Journal de Physique Lettres, 1977, 38 (2), pp.69-71. 10.1051/jphyslet:0197700380206900 . jpa-00231328

\section{HAL Id: jpa-00231328 https://hal.science/jpa-00231328}

Submitted on 1 Jan 1977

HAL is a multi-disciplinary open access archive for the deposit and dissemination of scientific research documents, whether they are published or not. The documents may come from teaching and research institutions in France or abroad, or from public or private research centers.
L'archive ouverte pluridisciplinaire HAL, est destinée au dépôt et à la diffusion de documents scientifiques de niveau recherche, publiés ou non, émanant des établissements d'enseignement et de recherche français ou étrangers, des laboratoires publics ou privés. 


\title{
CONTRIBUTION A L'ÉTUDE DE L'EFFET DE MAGNÉTOCONCENTRATION. UTILITÉ DU FORMALISME DE BOLTZMANN
}

\section{S. CRISTOLOVEANU}

\author{
Laboratoire d'Electronique, E.N.S.E.R., 23, avenue des Martyrs, 38031 Grenoble Cedex, France
}

(Reçu le 4 octobre 1976, révisé le 22 novembre 1976, accepté le 20 décembre 1976)

\begin{abstract}
Résumé. - Le phénomène de magnétoconcentration est fréquemment utilisé pour la détermination des paramètres de recombinaison dans les semiconducteurs. Nous montrons qu'une approche basée sur le formalisme hydrodynamique conduit à des erreurs très importantes, par rapport à une étude utilisant le formalisme de Boltzmann.
\end{abstract}

\begin{abstract}
The magnetoconcentration effect is often used for the determination of the recombination parameters of semiconductors. We show that a qualitative method of calculation such as the hydrodynamic formalism leads to large errors, in comparison with the Boltzmann formalism.
\end{abstract}

Dans cet article nous faisons une mise au point du phénomène de magnétoconcentration (déséquilibre thermodynamique de porteurs, apparaissant dans un barreau semiconducteur soumis à des champs électrique $\mathbf{E}_{x}$ et magnétique $\mathbf{B}_{z}$ croisés, selon la configuration de l'effet Hall, et dont l'une au moins des surfaces actives, parallèles à $\mathbf{E}_{x}$ et $\mathbf{B}_{z}$, n'est pas infiniment recombinante) $[1,2]$; cette mise au point s'appuie essentiellement sur le formalisme de Boltzmann : nous montrons qu'il apporte d'importantes corrections numériques aux résultats fournis par le formalisme hydrodynamique classique. Rappelons que l'effet de magnétoconcentration est largement utilisé pour la détermination de la durée de vie des porteurs et des vitesses de recombinaison en surface $[2,3,4]$, ainsi que pour la réalisation de divers dispositifs électroniques (capteurs magnétiques [5], détecteurs photoélectriques [6], etc.).

1. Equation de la magnétoconcentration. - Les relations de continuité des flux de porteurs conduisent à l'équation de la magnétoconcentration [1]. Notons que l'effet de magnétoconcentration (dont l'amplitude est proportionnelle à la densité de porteurs minoritaires) se détériore rapidement si on s'éloigne de l'état intrinsèque. Considérons donc l'équation de la magnétoconcentration (dont la solution est connue $[1,2,8])$ dans le cas d'un semiconducteur intrinsèque :

$$
D^{*} \frac{\partial^{2} n}{\partial y^{2}}+\frac{D^{*}}{l} \frac{\partial n}{\partial y}-\frac{\delta n}{\tau}=0
$$

où $\tau$ est la durée de vié des porteurs et $\delta n$ l'écart de la densité locale de porteurs $n(y)$ par rapport à la densité d'équilibre $n_{0}$; les coefficients ambipolaires $D^{*}, l$, font intervenir les mobilités $\mu_{\mathrm{n}, \mathrm{p}}$ des électrons et des trous, ainsi que les mobilités $\mu_{\mathrm{H}_{\mathrm{n}, \mathrm{p}}}$ de Hall, naturellement différentes des mobilités $\mu_{0_{n, p}}$ définies en absence d'induction magnétique. La solution analytique de l'éq. (1) est obtenue $[1,2,7]$ en associant des conditions aux limites sur les surfaces actives (dotées respectivement des vitesses de recombinaison $s_{1,2}$ ).

Il est bien connu [7] que les expressions $D^{*}, l$, déduites du formalisme de Boltzmann, sont différentes de celles déduites du formalisme hydrodynamique; nous rappelons dans le tableau A les expressions des coefficients $D^{*}, l$, calculées à partir des deux formalismes.

Nous allons montrer maintenant que l'étude de l'effet de magnétoconcentration à l'aide du formalisme de Boltzmann conduit à des résultats numériques notablement différents de ceux obtenus à l'aide du formalisme hydrodynamique [8]. En particulier, la détermination des durées de vie et des vitesses de recombinaison sur les surfaces à partir des expériences de magnétoconcentration exige l'utilisation du formalisme de Boltzmann; en guise d'illustration, nous traitons le cas de l'InSb $\left(\mu_{0_{\mathrm{n}}}^{2} B^{2} \gg 1\right.$ et $\mu_{0_{\mathrm{p}}}^{2} B^{2} \ll 1$ pour $0,4<B<5 \mathrm{~T}$ ), matériau sur lequel l'effet de magnétoconcentration a été largement étudié expérimentalement et théoriquement à l'aide du formalisme hydrodynamique $[3,4,9]$. 


\section{TABLEAU A}

Coefficients de l'équation de la magnétoconcentration pour diverses valeurs significatives de l'induction magnétique, obtenus à l'aide des formalismes de Boltzmann (dans l'hypothèse habituelle du libre parcours moyen constant) et hydrodynamique.

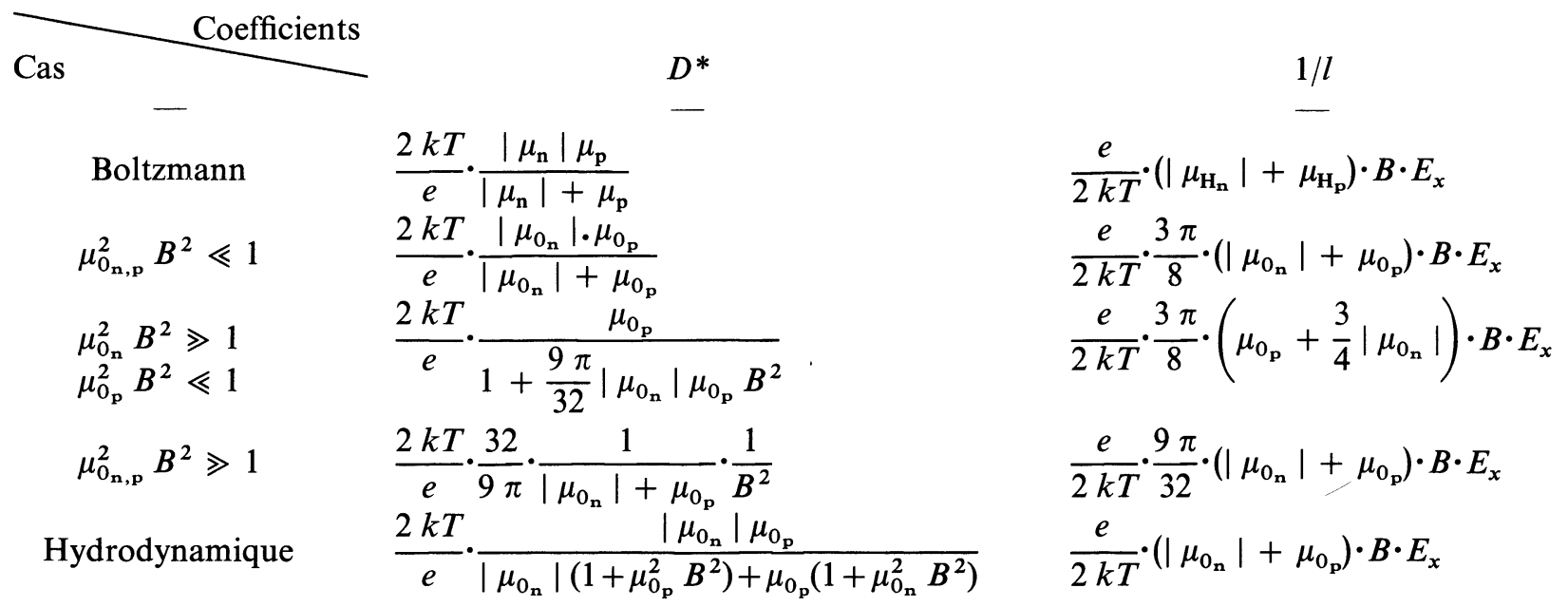

2. Analyse numérique de la magnétoconcentration dans l'InSb ; application à la mesure des paramètres de recombinaison. - Nous avons calculé, à l'aide d'un ordinateur, la densité relative moyenne de porteurs $\langle n\rangle / n_{0}$, proportionnelle au premier ordre à la conductivité électrique du barreau [8], en fonction de $\mathbf{E}, \mathbf{B}$ et pour divers paramètres de l'échantillon $\left(\tau, s_{1,2}\right.$, épaisseur $\left.b\right)$.

Les valeurs de $\langle n\rangle / n_{0}$ obtenues par les deux formalismes, pour une même durée de vie $\tau$, présentent des écarts allant jusqu'à $20 \%$ (Fig. 1) ; par ailleurs, une variation de $\tau$ entre 50 et 250 ns (domaine de variation possible de la durée de vie de l'InSb, à la température ambiante [4]) entraîne une variation de $\langle n\rangle / n_{0}$ de l'ordre de $50 \%$.

Du point de vue pratique, on constate sur ce graphique que, pour une conductivité du barreau déterminée expérimentalement (donc pour une valeur de $\left.\langle n\rangle / n_{0}\right)$, il correspond deux valeurs de $\tau$, selon le formalisme adopté, la différence pouvant facilement dépasser $100 \%$. Donc, la méthode (souvent utilisée $[3,4,9])$ de détermination de la durée de vie, s'appuyant sur l'analyse de l'influence de la magnétoconcentration sur la conductivité du barreau, ne peut être précise que si elle se fonde théoriquement sur le formalisme de Boltzmann.

Signalons qu'une telle précaution n'a pas été prise en considération dans les travaux précédents $[3,4,9]$.

De même, l'erreur sur la détermination des vitesses de recombinaison en surface est importante. A titre d'exemple, nous montrons dans la figure 2 que la mesure de la vitesse de recombinaison d'une surface décapée chimiquement $(s \rightarrow 0$, courbe 1$)$ ou disloquée mécaniquement $(s \rightarrow \infty$, courbe 2$)$, l'autre surface ayant subi le traitement contraire (ce qui conduit à une perturbation notable de la densité moyenne

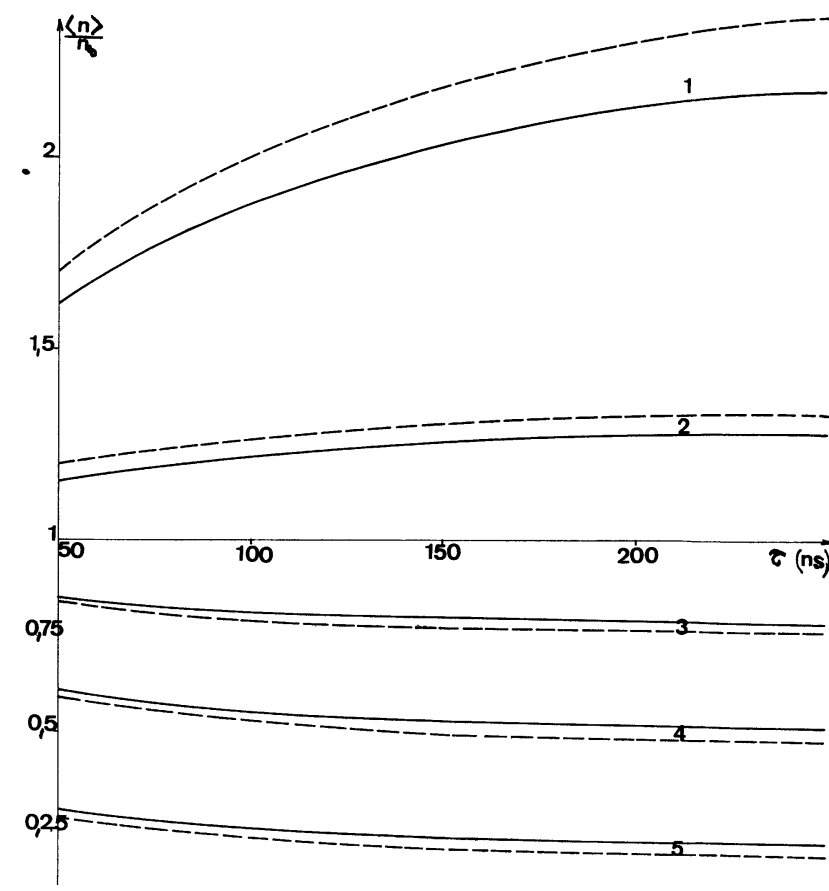

FIG. 1. - Densité relative moyenne de porteurs dans un barreau intrinsèque d'InSb, siège de l'effet de magnétoconcentration, en fonction de la durée de vie, pour différents champs électriques $\left(B_{z}=1,5 \mathrm{~T}\right.$; épaisseur du barreau $b=10 \mu \mathrm{m} ; s_{1}=1000 \mathrm{~m} \cdot \mathrm{s}^{-1}$ et $s_{2}=10 \mathrm{~m} . \mathrm{s}^{-1}$ ) : courbes selon le formalisme hydrodynamique ; -.--- courbes selon le formalisme de Boltzmann. (1) $E_{x}=900 \mathrm{~V} \cdot \mathrm{m}^{-1}$; (2) $300 ;$ (3) -300 ; (4) -900 ; (5) -2700 .

de porteurs), est affectée d'une incertitude pouvant dépasser $400 \%$.

Notons deux autres sources possibles d'erreurs, pouvant excéder $100 \%$, qui découlent de la présence de forts champs électriques $\left(E_{x}>10 \mathrm{~V} . \mathrm{cm}^{-1}\right.$ dans l'InSb) : 


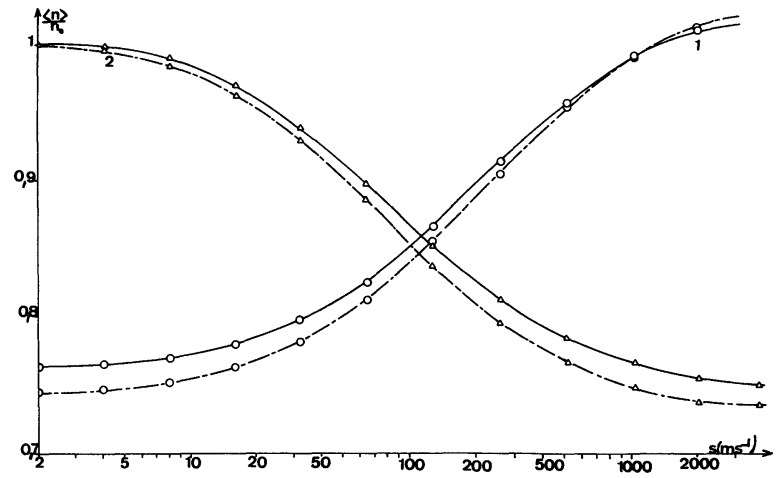

FIG. 2. - Densité relative moyenne de porteurs dans un barreau d'InSb, en fonction de l'état des surfaces $\left(B_{z}=1,5 \mathrm{~T}, b=20 \mu \mathrm{m}\right.$, $\left.E_{x}=300 \mathrm{~V} \cdot \mathrm{m}^{-1}, \tau=100 \mathrm{~ns}\right):-$ courbes selon le formalisme de Boltzmann ; - . - . - - courbes selon le formalisme hydrodynamique ; (1) $s_{1}=s ; s_{2}=1000 \mathrm{~m} . \mathrm{s}^{-1}$; (2) $s_{1}=5 \mathrm{~m} . \mathrm{s}^{-1}$; $s_{2}=s$.

* l'excédent de porteurs sur la surface enrichie devient de l'ordre de grandeur de la densité d'équilibre et la durée de vie ne peut plus être considérée comme constante ; la résolution de l'équation (1) est alors plus complexe [10];

* le terme de diffusion transversale n'est plus négligeable dans l'expression du courant électrique (c'est-à-dire que la conductivité du barreau n'est plus proportionnelle au nombre moyen de porteurs) [8]. La diffusion transversale entraîne une limitation du courant longitudinal $I_{x}\left(E_{x}\right)$.

L'importance de ces diverses corrections apparaît clairement dans la figure 2 . La courbe $\mathrm{H}$ a été obtenue théoriquement par Royer [3] à partir du formalisme hydrodynamique; la courbe B (dont l'allure est en parfait accord avec les courbes expérimentales obtenues par ailleurs $[3,4,9])$ a été tracée par nous pour le même jeu de paramètres, en utilisant le formalisme de Boltzmann et en tenant compte du terme de diffusion.

En conclusion, s'il était bien connu que le formalisme de Boltzmann renfermait plus de possibilités

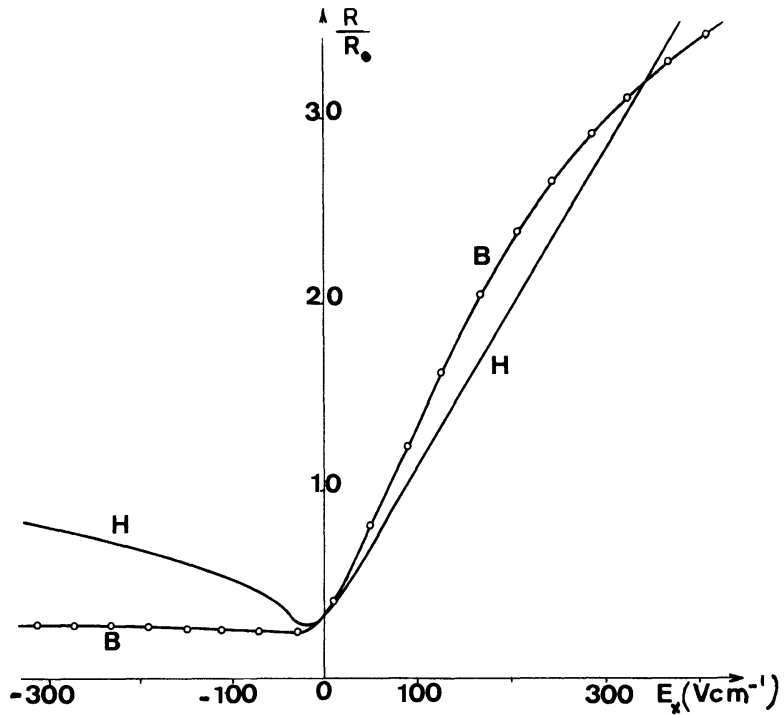

Fig. 3. - Comparaison des courbes théoriques de magnétorésistance anormale à partir des calculs de type hydrodynamique (courbe H) ou Boltzmann (courbe B). $\left(s_{1}=200 \mathrm{~m} \cdot \mathrm{s}^{-1}\right.$; $s_{2}=5000 \mathrm{~m} \cdot \mathrm{s}^{-1} ; b=10 \mu \mathrm{m} ; \tau=10 \mathrm{~ns} ; T=300 \mathrm{~K}$; $B_{z}=3 \mathrm{~T} ;\left|\mu_{0_{\mathrm{n}}}\right|=6,4 \mathrm{~m}^{2} \cdot \mathrm{V}^{-1} \cdot \mathrm{s}^{-1} ; \mu_{0_{\mathrm{p}}}=0,07 \mathrm{~m}^{2} \cdot \mathrm{V}^{-1} \cdot \mathrm{s}^{-1}$.)

pour décrire rigoureusement les phénomènes physiques et exprimer les grandeurs correspondantes, il était cependant admis que le formalisme hydrodynamique, offrant par sa simplicité des conditions d'analyse plus aisées, puisse conduire à des résultats voisins. Nous venons de voir, au contraire, qu'il est indispensable de s'appuyer sur le formalisme de Boltzmann, dans l'exploitation du phénomène de magnétoconcentration, pour la détermination de la durée de vie des porteurs et des vitesses de recombinaison en surface dans un semiconducteur, sous peine d'aboutir à des résultats numériques gravement entachés d'erreurs.

Je tiens à remercier Dr. P. Viktorovitch et Dr. G. Kamarinos pour les discussions approfondies que nous avons eues pendant la rédaction de ce travail.

\section{Bibliographie}

[1] Madelung, O., Tewordt, L., WelkeR, H., Z. Naturforsch. 10a (1955) 476.

[2] Chovet, A., Kamarinos, G., Revue Phys. Appl. 6 (1971) 345.

[3] ROYER, A., Thèse, Univ. Paris VI (1973).

[4] Preuss, E., Solid State Electron. 13 (1970) 173.

[5] Kamarinos, G., VikToRovitch, P., 6th European Solid State Device Research Conference, München (1976).
[6] Malyutenko, V. K., Teslenko, G. I. and Boiko, I. I., Sov. Phys. Semicond. 8 (1975) 1376.

[7] Pikus, G. E., Sov. Phys., Techn. Phys. 1 (1956) 17.

[8] Cristoloveanu, S., Thèse, I.N.P.G. (1976).

[9] Fujisada, H., J. Appl. Phys. 45 (1974) 3530.

[10] Chovet, A., Phys. St. Sol. (a) 28 (1975) 633. 